\title{
Soluble Epoxide Hydrolase: Potential Target for Inflammation and Inflammation-Driven Cancer
}

Chao Lou, Liping Zhang, Xiaobing Wang, Xiaoping Ma, Cuiyun Qin, Wei Li, Ting Jia, Qing Nan and Rong Qiang *

Department of Genetics, Northwest Women's and Children's Hospital, Xi'an, PR China

*Corresponding author: Rong Qiang, Department of Genetics, Northwest Women's and Children's Hospital, Xi'an, PR China, Tel: +862987234790; E-mail: qiangrongshx@126.com

Received date: Feb 28, 2017; Accepted date: May 16, 2017; Published date: May 22, 2017

Copyright: () 2017 Lou C, et al. This is an open-access article distributed under the terms of the Creative Commons Attribution License, which permits unrestricted use, distribution, and reproduction in any medium, provided the original author and source are credited.

\begin{abstract}
Arachidonic acid can be catalyzed by three different kind of metabolizing enzyme: cyclooxygenase (COX), lipoxygenase ( $L O X)$ and/or cytochrome P450 (CYP), and they produce prostaglandins, monohydroxys, leukotrienes and epoxyeicosanoids respectively. Through the cytochrome P450 pathway, arachidonic acid can be converted to two kinds of eicosanoid acids: epoxyeicosanoids acid (EET) by cytochrome P450 and hydroxyeicosatetraenoic acids (HETEs) formed by CYP a-oxidases.
\end{abstract}

Keywords: Cyclooxygenase; Lipoxygenase; Cytochrome P450

\section{Introduction}

Arachidonic acid can be catalyzed by three different kind of metabolizing enzyme: cyclooxygenase $(C O X)$, lipoxygenase $(L O X)$ and/or cytochrome $\mathrm{P} 450(C Y P)$, and they produce prostaglandins, monohydroxys, leukotrienesand epoxyeicosanoids respectively. Through the cytochrome P450 pathway, arachidonic acid can be converted to two kinds of eicosanoid acid: epoxyeicosanoids acid (EET) by cytochrome $\mathrm{P} 450$ and hydroxyeicosatetraenoic acids (HETEs) formed by CYP $\alpha$-oxidases $[1,2]$. EET is synthesized in the endothelium cells and plays a role as an endothelium-dependent hyperpolarizing factor (EDHF) under certain conditions in circulation [3]. EETs have diverse biological functions and have been found to play an important role in modulating hypertension and following renal damage, acute inflammation and inflammatory pain [4-10] which are essential for physiologic and pathologic processes in the body. Soluble epoxide hydrolase $(\mathrm{sEH})$ is an enzyme that metabolizes endogenous and/or exogenous epoxide-containing compounds to their corresponding diols and/or glycols by catalyzing the addition of water to the epoxide moiety.

Soluble epoxide hydrolase ( $\mathrm{sEH}$ ) is an enzyme that metabolizes endogenous and/or exogenous epoxide-containing compounds to their corresponding diols and/or glycols by catalyzing the addition of water to the epoxide moiety. Epoxide hydrolase are a family of enzymes that convert the epoxide group of compounds diols by adding water. According to their location, substrate and species, the epoxide hydrolase family is divided into 6 different subtypes: mammalian sEH, plant $\mathrm{sEH}$, microsomal epoxide hydrolase, hepoxilin hydrolase, insect juvenile hormone epoxide hydrolase, and leukotriene A4 hydrolase $[11,12]$. Mammalian sEH is the most important subtype because of its function in the metabolism of EETs. The human $\mathrm{sEH}$ is encoded by the $E P H X-2$ gene. $E P H X-2$ is located on chromosome 8p21-p12, nearly $45 \mathrm{~kb}$ in length and consists of 19 exons. sEH protein is 555 amino acids and has both an $\mathrm{N}$-terminal phosphatase activity that catalyzes the lipid backbone and a C-terminal [13]. The catalytic mechanism involves formation of a covalent alkylenzyme ester intermediate as a result of nucleophilic attack by Asp333. This is subsequently hydrolyzed with assistance of the general base His523 in a charge relay with Asp495 to yield the vicinal diol product. The C-terminal domain is an $\alpha / \beta$-hydrolase fold structure and is responsible for the epoxide hydrolase activity, and the substrates include both saturated and unsaturated fatty acid epoxide, especially the EETs [14,15]. sEH protein structure is complicated. The $\mathrm{x}$-ray crystal structures of murine sEH and human sEH show that most lipid epoxide substrates and inhibitors bond to the middle of an "L"-shaped hydrophobic tunnel in the sEH structure [16]. sEH protein is distributed in different human organs and tissues, such as the liver, colon, prostate, lymph node. According to data, the subcellular localization is cell-specific, in human hepatocytes and renal proximal tubules; $\mathrm{sEH}$ is both cytosolic and peroxisomal, but is exclusively cytosolic in other sEH-containing cells such as pancreatic islet cells, intestinal epithelium, anterior pituitary cells, adrenal gland, endometrium, lymphoid follicles, prostate ductal epithelium, alveolar wall, and blood vessels [17].

The CYP epoxygenases that synthesize EETs primarily consist of CYP 2C and CYP 2J classes, produce four different EETs (5,6-,8,9-, 11,12 - and 14,15-EET) by adding an epoxide group to one of the four double bonds of arachidonic acid. The CYP2C9 and CYP2J2 are mostly expressed endothelial cells. Each EETs regioisomers is composed by two EET isomers, because the epoxide group can attach at each of the double bonds in two different configurations, producing $\mathrm{R} / \mathrm{S}$ and $\mathrm{S} / \mathrm{R}$ enantiomers of each EET regioisomer [18]. EETs can be found in organs such as liver, kidney, heart and brain, and can also be detected in blood and urine. The CYP enzymes are located in the endoplasmic reticulum in different cells, and the main products in many cases are 11,12- and 14,15-EET. Endothelial cells can express and are the main source of EETs. When stimulated by bradykinin, methacholine, shear stress or ischemia reperfusion, EET production increases many times and produce a biological change in the vascular system [19-21]. CYP 2C is an endothelium-derived hyoerpolarizing factor synthase, whose major products are 11,12-EETs, CYP 2C and 11,12-EETs, and can both be induced by cyclic stretch. The EETs can be esterified into the sn-2 position of phospholipids by phospholipase A2 and released after stimulation from outside. The main EET catabolic pathway is converted to the corresponding dihydroxyeicosatrienoic acids (DHET) by sEH, and DHETs biological 
activity drops greatly compared to EETs. This enzyme utilizes 8,9-, $11,12-$, and 14,15-EET and 5,6-EET as substrate and produce different products. A 16-carbon epoxy-fatty acid accumulates when either 11,12 - or 14,15-EET undergoes partial -oxidation. A 22-carbon product is formed from 11,12- and 14,15-EET by chain elongation. A methyl-terminal hydroxyl group can also be inserted into 8,9-, 11,12-, and 14,15-EET by CYP-oxidases [18,20].

EETs have several physiologic functions including vasodilation, anti-inflammation effects, angiogenesis, cell proliferation, neuron and somatotrophs hormone release, $G$ protein signaling, ion channel activity, and a variety of effects associated with modulation of NFkB $[9,10,21,22]$. EETs can produce vasodilation of small vascular beds in many different organs including the heart, intestine, and kidney. The mechanism including hyperpolarization on the vascular smooth muscle and endothelium by EETs, or EETs directly activate the hyperpolarization and vaso-relaxation by forming a $\mathrm{Ca}^{2+}$ signaling complex. The EETs also activate $\mathrm{K}^{+}$and $\mathrm{Na}^{+}$channels through the control of PI3K signaling pathway [23-27]. The EETs can stimulate the angiogenesis of blood vessel through PI3K, MAPK pathway by changing the expression of P27, cyclin D1, JNK, CREBP and COX-2. EETs stimulate the proliferation of renal tubular and mesangial cells by activating Src and PI3K kinase pathway [26,27]. In the endothelium, EETs inhibit the cytokine-induced inflammatory response, enhance fibrinolysis, and increase $\mathrm{Ca}^{2+}$ channel open. EETs possess antiinflammatory properties and can attenuate monocyte infiltration. 11, 12-EET can inhibit IKK-mediated phosphorylation of IB, maintaining NF-B in an inactive state $[9,10]$. The anti-inflammatory effects of EETs were found to be associated with inhibition of leukocyte adhesion to the vascular wall by downregulation of transcription factor NFkB. Because EETs can be catalyzed to DHETs by sEH and DHETs lose the EETs biological activity and functions by adding water group, many EETs functions were decrease after the conversion. Therefore, sEH become a molecular target in treatment with hypertension and inflammation.

Increased expression of $\mathrm{sEH}$ has been found in hypertension animals. In angiotensin II (Ang II) hypertension rat model, sEH expression increased in renal microvessels and renal cortical tissue. In spontaneously hypertensive rats, expression of $\mathrm{sEH}$ increased at a prehypertensive stage ( $3 \mathrm{wk}$ of age) and hypertensive stage (9 wk of age) [28]. Not only sEH, but DEHT, the production of EETs hydrolysis, was also found increasing in Ang II hypertension rats model. Based on this, inhibition of $\mathrm{sEH}$ became a novel approach for treatment of hypertension. sEH inhibitors such as 1-adamantyl-3-(12aminododecyl) urea (AADU), 1-cyclohexyl-3-dodecylurea (CDU), 12(3-cyclohexyl-ureido) dodecanoic acid (CUDA), 12-(3-adamantan-1yl-ureido) dodecanoic acid (AUDA) and 1471 have been broadly used in experimental research and clinical treatment of hypertension. Olearczyk et al. the used mesenteric resistance arteries model, and found that AUDA can release the hypertension in rats model induced by thromboxane A2 agonist U46619 and induced arteries vasodilation by extending on vascular smooth muscle. AUDA can significantly lower blood pressure in both high-salt and normal-salt hypertension rats models, and the drop in blood pressure is connected with urinary epoxide-to-diol ratios [29]. At the same time, AUDA protects the renal damage following the hypertension. Loch $\mathrm{D}$ et al. [30] reported that another sEH inhibitor $\mathrm{N}$-adamantyl-N'-dodecylurea (ADU) prevent deoxycorticosterone acetate (DOCA)-salt developed rats hypertension, hypertrophy, perivascular and interstitial fibrosis, and endothelial dysfunction. Administration of ADU prevented the further increase in systolic blood pressure and left-ventricular wet weight and normalized endothelial function.

sEH has a close connection with pre-inflammation, inflammatory process and inflammation driven pain. Because metabolizing of arachidonic acid is controlled by COX, LOX, and CYP pathways, many of these enzymes generate reactive oxygen species which enhance the inflammatory processes or are inflammatory cytokine themselves. The interaction between EETs and these inflammation medias, such as PGE2, iNOS, COX-1 and COX-2 is complexed. Node K et al. [9] reported that EETs prevented the amplification of an inflammatory event by inhibition of transcription factor NF- B and I B kinase. Because translocation of NF-B from cytoplasm to the nucleus will make resident macrophages produce proinflammatory proteins, such as TNF- $\alpha$, IL6, iNOS, and COX-2, therefore, EETs can also prevent the surface expression of cell adhesion molecules, which would further suppress infiltration of leukocytes and increase proinflammatory mediators, including cytokines and oxylipins. EETs not only modulate epoxygenase metabolism but also have a connection with LOX and COX metabolism pathways, which affects the COX-2 and 5-LOX [4]. An increase in EETs caused by sEH inhibitor has little effect on the constitutive COX-1 or even COX-2 in plasma, but can greatly decrease the expression amount of induced COX-2 and its associated metabolites which lead to an increase in symptoms associated with severe inflammation. EETs down regulate the transcription of COX-2, and might shift arachidonic acid into the LOX pathway, thus increasing proinflammatory eicosanoids [31]. The metabolism production of 5-LOX is the 5-hydroperoxyeicosatetraenoic acid. It can be further catalyzed to 5-HETE and leukotrienes, and ultimately, lipoxins. Inflammatory resolution is promoted by the production of lipoxins, which suppress leukocyte recruitment, neutrophil adherence to endothelial and epithelial cells, and macrophage clearance of apoptotic neutrophils [4]. Many inflammatory mediators such as histamine, serotonin, kinins, cytokines and arachidonic acid metabolites, can stimulate the peripheral nerve distal fibers and produce inflammatory pain. Arachidonic acid is crucial in this process and has been studied for a long time, but previous research was focused on the products of COX and LOX enzyme pathway of arachidonic acid, such as prostaglandins (PGs) and leukotrienes [32-34]. The products of cytochrome P450 (CYP) enzyme pathway were also found to have a close relation with inflammatory pain, especially the EETs. Two sEH inhibitors- 12-(3-adamantan-1-ylureido)-dodeca-noic acid butyl ester (AUDA-BE) and 1-adamantan-3(5-(2-(2-ethylethoxy) ethoxy)pentyl)urea (IK 950) can suppress the thermal hyperalgesia and mechanical allodynia induced by LPS in rats in the inflammatory pain model and EETs can also distinctly decrease thermal hyperalgesia in this model. Actually, the pain relief dependents on the concentration of EETs no matter what kind of sEH inhibitor it is.

Therefore, sEH inhibitors are very important in treatment and prevention of in inflammation and study of $\mathrm{sEH}$ inhibitors provides a strong foundation for the development of antiinflammatory therapeutics. Adamantyl alkyl urea-based sEH inhibitors increase fatty acid epoxides, indirectly reduce the production of NO, cytokines, and proinflammatory lipid mediators, and can enhance the production of inflammatory resolution by promoting lipoxin A4 production. In LPSinduced inflammation, the $\mathrm{NO}$ metabolites nitrite $\left(\mathrm{NO}^{-2}\right)$ and nitrate $\left(\mathrm{NO}^{-3}\right)$ were elevated in the plasma, sEH inhibitors A12-(3adamantan-1-yl-ureido)dodecanoic acid butyl ester (AUDA-BE) can markedly reduce NO production, and at the same time, suppress the induced iNOS expression level in the liver [4]. In addition, COX-2 
protein levels in liver, which are an important sign of proinflammatory gene expression, were also suppressed by the $\mathrm{sEH}$ inhibitor. Because of the observed decline in hepatic COX-2 expression, AUDA-BE decreas the following COX-2 production such as prosta-5,13-dien-1-oic acid, 9,15-dihydroxy-11-oxo-, (5Z,9,13E,15S) (PGD2) and prosta-5,13dien-1-oic acid, 11,15-dihydroxy-9-oxo-, (5Z,11,13E,15S) (PGE2), 9,11,15S-trihydroxythromba-5Z,13E-dien-1-oic acid (TXB2) and 6oxo-9,11,15S-trihydroxy-prost-13-en-1-oic acid (6-keto-PGF1) at the same time $[4,31]$. From the research data, the mechanism and function of sEH's decrease of the induction of COX-2 is that EETs down regulate the expression of COX-2 and decrease the COX-2 induced inflammatory cytokine and chemokine. Schmelzer KR et al. [4] reported that AUDA-BE can greatly diminish lipopolysaccharide (LPS)-induced mortality, systemic hypotension, and tissue injury in C57BL_6 mice. At the same time, AUDA-BE can also decrease plasma levels of proinflammatory cytokines and nitric oxide metabolites while promoting the formation of lipoxins, thus supporting inflammatory resolution. The sEH inhibitor AUDA-BE can also inhibit tobacco smoke-induced inflammation by significantly decreasing neutrophils, alveolar macrophages, and lymphocyte cell numbers in bronchoalveolar lavage [8]. sEH inhibitors have also been proven to reduce inflammatory pain and hyperalgesia in LPS induced inflammatory in rat model, the mechanism include increasing the EETs and suppressing the PG synthesis. Inhibition of sEH may become a promising therapeutic strategy to attain analgesia. Unlike the LOX/COX inhibitor, which acts directly to prevent synthesis of proinflammatory lipid mediators, the $\mathrm{sEH}$ inhibitors stabilize antiinflammatory oxylipins and elevate proresolution mediators. COX inhibitors can increase EET concentration. The combined use of COX inhibitors and $\mathrm{sEH}$ inhibitors can not only greatly reduce inflammatory metabolism, but also depress the side effects of COX inhibitors [31]. A combination of sEH inhibitor and EETs can enhance the inhibition of LPS induced inflammation more significantly than with the sEH inhibitors alone [31].

To the study further, $s E H$ gene knockout mouse model was used to find the role of $\mathrm{sEH}$ in the mammalian body. The systolic blood pressure in male sEH knockout mouse was lower compared than wildtype mice in both the absence and presence of dietary salt loading. In the acute systemic inflammation model, the sEH-null mice show a survival advantage over normal control [35]. In sEH-null mice, plasma levels of epoxy fatty acids were increased, fatty acid diols levels were decreased, and renal formation of epoxyeicosatrienoic and dihydroxyeicosatrienoic acids was markedly lower for versus wild-type mice [36,37]. However, in cardiac arrest (CA) and the resuscitation mouse model, the survival rate of sEH knockout mice model was significantly lower than wild-type, and reduced sEH levels or function reduce survival from cardiac arrest. This result is contrary to the previous hypothesis that $\mathrm{sEH}$ gene deletion improves survival and protect against ischemia/reperfusion injury [36]. To find subcellular location and genetic polymorphisms of $\mathrm{sEH}, \mathrm{sEH} \mathrm{cDNA}$ was cloned into vectors and transfected into different cell lines. Mullen et al. fused soluble epoxide hydrolase to green fluorescent protein, and found that $s E H$ is located in cytosolic and peroxisomal in mice and rats. Because of genetic polymorphisms, after screening the sEH cDNA sequence, different variant loci can be identified. Sandberg $M$ et al. used two different sEH cDNA vectors to transfect $\mathrm{CHO}$ and COS-7 cells transiently, and found that different allelic forms of human sEH have different enzymatic activity due to different epoxide exposures.

Arachidonic acid epoxides are involved in apoptosis, oncogenesis and cell proliferation. N-terminal phosphatase domain of $s E H$ hydrolyzes isoprenoid mono- and pyrophosphates, which in cell signaling and apoptosis. Enayetallah AE et al. [34] have proved that sEH expression in renal, hepatic malignant neoplasms and seminoma was significantly increased compared to surrounding non-neoplastic tissues or normal tissues. We know that EETs have a close relation with PI3K pathway, COX-2, NFkB, and inducible nitric oxide synthase (iNOS), which are very important in inflammation and inflammation induced carcinomas. LPS and TNF- $\alpha$ can induce the $s E H$ expression in different kinds of cells. LPS and TNF- $\alpha$ are known to stimulate activation of PI3K pathways in cells, and expression $\mathrm{sEH}$ may be controlled by PI3K pathway. Many malignances are developed from chronic inflammation, such as carcinomas in colon, prostate, liver, stomach and the lung. The relation between inflammation and cancer has been confirmed, but the mechanism is still unclear. The features of inflammation driven cancer include infiltration of white neutrophilic granulocyte, and involvement of cytokines and chemokines. Inflammation is a critical component of tumor progression, the inflammatory cells and cytokines have always been found in the tumor process, and may contribute to tumor proliferation, differentiation, and progression. Many cancers were found to rise from sites of infection, chronic irritation and inflammation. Coussens LM et al. [32] reported that tumor microenvironment, which is established by inflammatory cells themselves, is essential in the malignant process, building a good environment for tumor cells to proliferate, survive and migrate. Further, tumor cells can interact and select some of chemical signaling molecules of the immune system, such as selectins, chemokines and their receptors for invasion, migration and metastasis. Balkwill $\mathrm{F}$ et al. [33] also found that cancer susceptibility and development may be associated with functional polymorphisms of inflammatory cytokine genes, and deletion or inhibition of inflammatory cytokines inhibits development of experimental cancer. Acute inflammation may kill bacteria and viruses enhance the immune system, but the chronic inflammation will consume energy quickly, impair the immune system, and increase the abnormal proliferation. These insights are fostering new anti-inflammatory therapeutic approaches to cancer development. It is the reason why non-steroidal anti-inflammatory drugs-a cytokine and chemokine inhibitor are often used to treat of patients with cancer and for chemoprevention of cancer. PI3K pathway has been proved to be a bridge between inflammation and cancers. Our research is focused on the inflammatory bowel disease (IBD) (including ulcerative colitis UC, and Crohn's disease CD) and IBD-induced cancer. In IBD, IL-3, IL-6, IL-7, EPO and INF- $\lambda$ inhibit apoptosis of $\mathrm{T}$ cells through PI3K pathway. NFKB is very important in regulating immune response, IL-1, LPS and TNF- $\alpha$ can stimulate the expression of NFKB, then increase $\mathrm{NF \kappa B}$ induced inflammatory mediators and damage colon epithelium cells in IBD. Data show NFKB may play a key role in the pathologic process of IBD. IL-1, LPS and TNF- $\alpha$ can activate NFkB by PI3K pathway and increase activation of NFKB. IL-1 and TNF-a can also activate NFkB subunit p65 and p50 by stimulating AKT and then activating NFKB. PI3K pathway can also stimulate the expression of NFKB by the MAPK and Cot pathway. Activated NFKB is involved in the transcription of many enzyme that control the inflammatory process, such as COX-2, iNOS, and IL-8. PI3K pathway is also very important in the lymphocyte chemotaxis and homing, deletion of p110gamma and p110delta or suppression of PI3K pathway by Wortmannin to reduce the $\mathrm{B}$ lymphocyte moving to intestine and spleen lymphoid node. In PI3K $\lambda-/$ - colon inflammation model mice, concentration of many dysfunctional white neutil cell was found, IL-5 and C5a-induced chemotaxis of lymphocyte decreased greatly. Node $\mathrm{K}$ et al. [9] observed that EETs prevented leukocyte adhesion to the 
vascular wall by a mechanism involving inhibition of transcription factor NFKB. Another inflammatory factor -iNOS, is very important in the process of UC and overproduced in the colon of UC patients. Nitric oxide stress may play an important role in conversion from inflammation to cancer by stimulating abnormal proliferation of colon epithelium cells. Nitric oxide (NO) molecules can damage DNA structure and cause mutation of some oncogene and tumor suppressor genes. For example, the tumor suppressor gene p53 is frequently mutated in the non-cancerous colonic mucosa of patients with UC.

\section{Conclusion}

Much research has been done in elucidating the role of sEH in hypertension inflammation and inflammatory pain, but the relationship between inflammation driven cancer and $\mathrm{sEH}$ has never been studied. Expression of sEH was found in colon epithelial cells, and we find the expression of sEH in the colon epithelial cancer cell line is much higher than in immortalized normal colon epithelial cell line (our own data), but the role of sEH in cancer still not clear. Does $\mathrm{N}$-terminal phosphatase domain of sEH play an important role in the carcinogenesis of colon? sEH inhibitors can suppress the colon inflammation process, could it suppress the inflammation driven colon carcinogenesis? Can sEH inhibitors become a new colon cancer chemoprevention agent? This question still needs to be addressed and further work need to be done on this subject in the near future.

\section{References}

1. Brash AR (2001) Arachidonic acid as a bioactive molecule. J Clin Invest 107: $1339-1345$

2. Oliw EH (1994) Oxygenation of polyunsaturated fatty acids by cytochrome P450 monooxygenases. Prog Lipid Res 33: 329-354.

3. Sinal CJ, Miyata M, Tohkin M, Nagata K, Bend JR, et al. (2000) Targeted disruption of soluble epoxide hydrolase reveals a role in blood pressure regulation. J Biol Chem 275: 40504-40510.

4. Schmelzer KR, Kubala L, Newman JW, Kim IH, Eiserich JP, et al. (2005) Soluble epoxide hyrolase is a therapeutic target for acute inflammation. Proc Natl Acad Sci USA 102: 9772-9777.

5. Inceoglu B, Jinks SL, Schmelzer KR, Waite T, Kim IH, et al. (2006) Inhibition of soluble epoxide hydrolase reduces LPS-induced thermal hyperalgesia and mechanical allodynia in a rat model of inflammatory pain. Life Sci 79: 2311-2319.

6. Fleming I (2007) DiscrEET regulators of homeostasis: epoxyeicosatrienoic acids, cytochrome P450 epoxygenases and vascular inflammation. Trends Pharmacol Sci 28: 448-452.

7. Imig JD, Zhao X, Zaharis CZ, Olearczyk JJ, Pollock DM, et al. (2005) An orally active epoxide hydrolase inhibitor lowers blood pressure and provides renal protection in salt-sensitive hypertension. Hypertension 46: 975-981.

8. Smith KR, Pinkerton KE, Watanabe T, Pedersen TL, Ma SJ, et al. (2005) Attenuation of tobacco smoke-induced lung inflammation by treatment with a soluble epoxide hydrolase inhibitor. Proc Natl Acad Sci U S A 102: 2186-2191.

9. Node K, Huo Y, Ruan X (1999) Anti-inflammatory properties of cytochrome P450 epoxygenasederived eicosanoids. Science 285: 12761279.

10. Kozak W, Kluger MJ, Kozak A, Wachulec M, Dokladny K (2000) Role of cytochrome P-450 in endogenous antipyresis. Am J Physiol 279: 455-460.

11. Newman JW, Morisseau C, Hammock BD (2005) Their roles and interactions with lipid metabolism. Prog Lipid Res 44: 1-51.

12. Xiang F (2006) epoxide hydrolases: a novel target for the treatment of Hypertension. Car Drug Dis 1: 67-72.
13. Cronin A, Mowbray S, Dürk H, Homburg S, Fleming I, et al. (2003) The $\mathrm{N}$-terminal domain of mammalian soluble epoxide hydrolase is a phosphatase. Proc Natl Acad Sci U S A. 100: 1552-1557.

14. Falck JR, Reddy LM, Reddy YK, Bondlela M, Krishna UM, et al. (2003) soluble epoxide hydrolase is a phosphatase. Bioorg Med Chem Lett 13: 4011-4014.

15. Sasaki M, Ostanin D, Elrod JW, Oshima T, Jordan P, et al. (2003) soluble epoxide hydrolase. Am J Physiol 284: 422-442.

16. Gomez GA, Morisseau C, Hammock BD, Christianson DW (2006) Human soluble epoxide hydrolase: structural basis of inhibition by 4-(3cyclohexylureido)-carboxylic acids. Protein Sci 15: 58-64.

17. Enayetallah AE, French RA, Barber M, Grant DF (2006) Cell-specific subcellular localization of soluble epoxide hydrolase in human tissues. J Histochem Cytochem 54: 329-335.

18. Gauthier KM, Deeter C, Krishna UM, Reddy YK, Bondlela M, et al. (2002) 14,15-Epoxyeicosa-5(Z)-enoic acid: a selective epoxyeicosatrienoic acid antagonist that inhibits endothelium-dependent hyperpolarization and relaxation in coronary arteries. Circ Res 90: 1028-1036.

19. Chen JK, Wang DW, Falck JR, Capdevila J, Harris RC (1999) Transfection of an active cytochrome P450 arachidonic acid epoxygenase indicates that 14,15-epoxyeicosatrienoic acid functions as an intracellular messenger in response to epidermal growth factor. J Biol Chem 274: 4764-4769.

20. Collins XH, Harmon SD, Kaduce TL, Berst KB, Fang X, et al. (2005) Oxidation of 20-hydroxyeicosatetraenoic acid (20-HETE) in cerebral microvascular smooth muscle and endothelium by alcohol dehydrogenase. J Biol Chem 280: 33157-33164.

21. Spector AA, Norris AW (2007) Action of epoxyeicosatrienoic acids on cellular function. Am J Physiol Cell Physiol 292: 996-1012.

22. Inceoglu B, Schmelzer KR, Morisseau C, Jinks SL, Hammock BD (2007) Soluble epoxide hydrolase inhibition reveals novel biological functions of epoxyeicosatrienoic acids (EETs). Prostaglandins Other Lipid Mediat 82: 42-49.

23. Dumoulin M, Salvail D, Gaudreault SB, Cadieux A, Roussseau E (1998) Epoxyeicosatrienoic acids relax airway smooth muscles and directly activate reconstituted KCa channels. Am J Physiol Lung Cell Mol Physiol 275: L423-L431.

24. Earley S, Heppner TJ, Nelson MT, Brayden JE (2005) TRPV4 forms a novel $\mathrm{Ca} 2+$ signaling complex with ryanodine receptors and BKCa channels. Circ Res 97: 1270-1279.

25. Lu T, Hong MP, Lee HC (2005) Molecular determinants of cardiac KATP channel activation by epoxyeicosatrienoic acids. J Biol Chem 280: 1909719104.

26. Weintraub NL, Spector AA, Lee HC (2001) Activation of ATP-sensitive K + channels by epoxyeicosatrienoic acids in rat cardiac ventricular myocytes. J Physiol 537: 881-827.

27. Delerive P, Martin-Nizard F, Chinetti G, Trottein F, Fruchart JC, et al. (1999) Peroxisome proliferator-activated receptor activators inhibit thrombin-induced endothelin-1 production in human vascular endothelial cells by inhibiting the activator protein-1 signaling pathway. Circ Res 85: 394-402.

28. Seubert J, Yang B, Bradbury JA, Graves J, Degraff LM, et al. (2004) Enhanced postischemic functional recovery in CYP2J2 transgenic hearts involves mitochondrial ATP-sensitive K+ channels and p42/p44 MAPK pathway. Circ Res 95: 506-514.

29. Seubert JM, Xu F, Graves JP, Collins JB, Sieber SO, et al. (2005) Differential renal gene expression in prehypertensive and hypertensive spontaneously hypertensive rats. Am J Physiol Renal Physiol 289: 552-561.

30. Olearczyk JJ, Field MB, Kim IH, Morisseau C, Hammock BD, et al. (2006) Substituted adamantyl-urea inhibitors of the soluble epoxide hydrolase dilate mesenteric resistance vessels. J Pharmacol Exp Ther 318: 1307-1314.

31. Loch D, Hoey A, Morisseau C, Hammock BO, Brown L (2007) Prevention of Hypertension in DOCA-Salt Rats by an Inhibitor of Soluble Epoxide Hydrolase. Cell Biochem Biophys. 47: 87-98. 
Citation: Lou C, Zhang L, Wang X, Ma X, Qin C, et al. (2017) Soluble Epoxide Hydrolase: Potential Target for Inflammation and InflammationDriven Cancer. J Carcinog Mutagen 8: 294. doi:10.4172/2157-2518.1000294

Page 5 of 5

32. Inceoglu B, Schmelzer KR, Morisseau C, Jinks SL, Hammock BD (2007) Soluble epoxide hydrolase inhibition reveals novel biological functions of epoxyeicosatrienoic acids (EETs). Prostaglandins Other Lipid Mediat 82: 42-49.

33. Coussens LM, Werb Z (2002) Inflammation and cancer. Nature 420 860-867.

34. Balkwill F, Mantovani A (2001) Inflammation and cancer: back to Virchow? Lancet 357: 539-545.

35. Enayetallah AE, French RA, Grant DF (2006) Distribution of soluble epoxide hydrolase, cytochrome P450 2C8, 2C9 and 2J2 in human malignant neoplasms. J Mol Histol 37: 133-141.
36. Hutchens MP, Nakano T, Dunlap J, Traystman RJ, Hurn PD, et al. (2007) Soluble epoxide hydrolase gene deletion reduces survival after cardiac arrest and cardiopulmonary resuscitation. Resuscitation 27: 8 .

37. Luria A, Weldon SM, Kabcenell AK, Ingraham RH, Matera D, et al. (2007) Compensatory mechanism for homeostatic blood pressure regulation in Ephx2 gene-disrupted mice. J Biol Chem 282: 2891-2898. 